\title{
1 Investigations into the emergent properties of gene-to- 2 phenotype networks across cycles of selection: A case study of 3 shoot branching in plants
}

\author{
4 Owen M. Powell ${ }^{\mathrm{a}, \mathrm{b},{ }^{*}}$, Francois Barbier ${ }^{\mathrm{b}, \mathrm{c}}$, Kai P. Voss-Fels ${ }^{\mathrm{d}, \mathrm{e}}$, Christine Beveridge ${ }^{\mathrm{b}, \mathrm{c}}$, Mark \\ 5 Cooper ${ }^{a, b}$
}

6 a University of Queensland, Queensland Alliance for Agriculture and Food Innovation, Centre for

7 Crop Science, St Lucia, QLD, Australia.

$8{ }^{b}$ ARC Centre of Excellence for Plant Success in Nature and Agriculture, University of

9 Queensland, St Lucia, QLD, Australia.

$10{ }^{\mathrm{C}}$ University of Queensland, School of Biological Sciences, St Lucia, QLD, Australia.

11 d University of Queensland, Queensland Alliance for Agriculture and Food Innovation, Centre for

12 Animal Science, St Lucia, QLD, Australia.

$13{ }^{e}$ Hochschule Geisenheim University, Department of Grapevine Breeding, Geisenheim, Germany.

15 Corresponding Authors:

16 * Owen M. Powell

17 Email: o.powell2@uq.edu.au

18 Author Contributions: OMP and MC conceived the study and interpreted the results. OMP

19 developed the gene-to-phenotype network, developed the in-silico selection experiments,

20 performed the analyses, and wrote the manuscript. FB provided input on the shoot branching

21 model, helped interpret the results, and wrote parts of the introduction. KVF developed a previous

22 iteration of the G2P network. CB provided input on the shoot branching model and helped

23 interpret the results. All authors read, refined, and approved the final manuscript.

24 Competing Interest Statement: The authors declare they have no competing interests.

25 Keywords: shoot architecture, , networks, epistasis, hormones, genetic canalization, cryptic

26 genetic variation..

This file includes: 


\section{Abstract}

33 Predictive breeding is now widely practised in crop improvement programs and has accelerated

34 selection response (i.e., the amount of genetic gain between breeding cycles) for complex traits.

35 However, world food production needs to increase further to meet the demands of the growing

36 human population. The prediction of complex traits with current methods can be inconsistent

37 across different genetic, environmental, and agronomic management contexts because the

38 complex relationships between genomic and phenotypic variation are not well accounted for.

39 Therefore, developing gene-to-phenotype network models for traits that integrate the knowledge

40 of networks from systems biology, plant and crop physiology with population genomics has been

41 proposed to close this gap in predictive modelling. Here, we develop a gene-to-phenotype

42 network for shoot branching, a critical developmental pathway underpinning harvestable yield for

43 many crop species, as a case study to explore the value of developing gene-to-phenotype

44 networks to enhance understanding of selection responses. We observed that genetic

45 canalization is an emergent property of the complex interactions among shoot branching gene-to-

46 phenotype network components, leading to the accumulation of cryptic genetic variation, reduced

47 selection responses, and large variation in selection trajectories across populations. As genetic

48 canalization is expected to be pervasive in traits, such as grain yield, that result from interactions

49 among multiple genes, traits, environments, and agronomic management practices, the need to

50 model traits in crop improvement programs as outcomes of gene-to-phenotype networks is

51 highlighted as an emerging opportunity to advance our understanding of selection response and

52 the efficiency of developing resilient crops for future climates. 


\section{Introduction}

54

Due to an increasingly harsh and unpredictable climate, improving the consistency and scope of predictions for crop performance is crucial for global agriculture to meet the challenge of feeding a global population of $10+$ billion people (Reynolds et al., 2021). Current prediction methods used in crop breeding assume a simplified linear relationship between genotype and phenotypes (Falconer and Mackay, 1996; Meuwissen et al., 2001; Cooper et al., 2014; Walsh and Lynch, 2018; Gianola, 2021), thus limiting the realized selection response achieved by many crop improvement programs (Kholová et al., 2021). Although this simplified genotype-tophenotype relationship (G2P map) is sufficient to successfully model the average selection trajectory of large populations (Cooper et al., 2014), this approach captures only a subset of all performance outcomes, potentially leading to misalignments between predicted performance and realized performance in the field. Such simplified G2P relationships can hinder accurate predictions for crop performance in specific management and environment combinations (Kholová et al., 2021).

Developing G2P network models that integrate the knowledge of networks from systems biology and physiology with population genomics may improve modelling of the genotype-tophenotype relationship for many complex traits (Benfey and Mitchell-Olds, 2008; Marjoram et al., 2014; Marshall-Colon et al., 2017; Eshed and Lippman, 2019; Cooper et al., 2020). For crop improvement programs, the detected interactions in network models can unmask existing genetic variation or identify intermediate traits that can increase the selection accuracy and efficiency of developing novel crop varieties and hybrids. Applying the framework of the "breeders equation" (Falconer and Mackay, 1996; Paixão and Barton, 2016; Walsh and Lynch, 2018), the contributions of the genetic interactions (epistasis) to total genetic variation must be converted into additive genetic variation to deliver a sustainable selection response in breeding programs (Technow et al. 2021). A strong theoretical understanding exists of the importance of epistasis for selection response when based on G2P models without molecular network models (Paixão and 
80 Barton, 2016; Walsh and Lynch, 2018). However, relevant knowledge of selection response for

81 G2P models based on networks operating over biological scales (Marshall-Colon et al., 2017;

82 Hammer et al., 2019; Wu et al., 2019; Tardieu et al., 2020) is lagging due to the lack of

83 adequately characterized empirically-based examples.

Here, we use axillary bud outgrowth, a primary driver of shoot branching, as a case study

of an agronomically important, well-characterized and empirically described network (Bertheloot et al., 2020). The core structure of this network is largely conserved across herbaceous model plants and divergent crops. It involves different endogenous signals interacting with each other to regulate shoot branching. Hormones including auxin, cytokinins and strigolactones play a crucial role in regulating this process (Domagalska and Leyser, 2011; Barbier et al., 2019). The growing shoot apex produces auxin, which travels downwards in the stem, inhibiting cytokinin synthesis and accumulation and promoting strigolactone synthesis. Cytokinins and strigolactones induce and repress bud outgrowth, respectively. Sugar availability is a determining factor for plant growth and development, including shoot branching (Barbier et al., 2019; Fichtner et al., 2021). Axillary buds require a source of sugar to grow out, and the strong demand for sugar by the shoot apex inhibits axillary bud outgrowth (Mason et al., 2014; Barbier et al., 2019). During shoot branching, sugars were reported to promote cytokinin synthesis and inhibit strigolactone perception (Barbier et al., 2019; Bertheloot et al., 2020; Salam et al., 2020; Patil et al., 2021). Despite the detailed knowledge of molecular physiological mechanisms, translating these discoveries into breeding outcomes is still a challenge.

Therefore, the objectives of this study were threefold: (1) to extend the Bertheloot et al. (2020) model of the shoot branching network to include genetic variation for nodes of the network; (2)

102 apply quantitative genetic methods (Falconer and Mackay, 1996; Walsh and Lynch 2018) to 103 undertake in silico investigations of important G2P properties of the extended shoot branching network model that can influence breeding outcomes; and (3) develop hypotheses of the

105 expected selection trajectories for levels of the branching network nodes and branching trait 106 outcomes for experimental investigation. Applying the framework developed to link quantitative 
107 genetic models for trait genetic variation with crop growth models to model plant responses to 108 environmental variation, outlined in Cooper et al. (2020), we created a shoot branching G2P 109 network (Fig. 1) underpinned by genomic variation to demonstrate how variation from interactions 110 in network-based G2P models is translated into selection responses for complex traits. In-silico 111 selection experiments were performed on a large, segregating plant population to quantify direct 112 (time to bud outgrowth) and indirect (intermediate traits; hormones and sucrose) selection 113 responses. The results are discussed in terms of practical implications for developing G2P 114 models to accelerate crop genetic improvement within established and future crop improvement 115 programs.

\section{Materials and Methods}

\section{Overview}

121 We created a gene-to-phenotype (G2P) network model for bud outgrowth by connecting a 122 published, empirical shoot branching network (Bertheloot et al., 2020) to underlying allelic 123 variation in the genome. The shoot branching network models phenotypic variation for the trait 124 "time to bud outgrowth" as the outcome of the intermediate traits, auxin, cytokinins, 125 strigolactones, and sucrose, and their interactions (Fig.1). We simulated the additive genetic 126 effects of 10 non-pleiotropic, causal genetic loci for each intermediate trait. The additive genetic 127 effects of the 10 causal genetic loci determined the additive genetic values for each intermediate 128 trait for individual genotypes. . These additive genetic values replaced the synthesis term of the 129 intermediate traits (hormones and sucrose), in the differential equations provided by Bertheloot et 130 al. (2020) which were used to calculate the levels for each intermediate trait $(g)$. Therefore, the 131 trait under selection, time to bud outgrowth $\left(\boldsymbol{y}_{\boldsymbol{B}}\right)$, can be viewed as a function $\mathrm{F}$ of the levels of 132 the genotype-dependent intermediate traits $(\boldsymbol{g})$ and a random error term $(\boldsymbol{e})$. 


$$
y_{B o}=F\left(g_{A}, g_{C K}, g_{S L}, g_{S U C}\right)+e
$$

135 To quantify response to selection, we performed in-silico divergent selection experiments for 136 increased and decreased levels of $\boldsymbol{y}_{\boldsymbol{B}}$ over 30 selection cycles. The results presented are 137 generated from 100 replicates of the in-silico selection experiment. Code for the shoot branching 138 network, genetic simulations and figures can be accessed in the following repository: 139 https://github.com/powellow/ GeneticCanalizationOfG2PNetworks.

\section{Description of the Empirical Shoot Branching Model}

Bertheloot et al. (2020) used experimental data to describe phenotypic variation for time

144 to bud outgrowth within a shoot branching network as a system of differential equations. The 145 shoot branching network takes levels of auxin and sucrose as inputs, calculates cytokinins, 146 strigolactones and signal integrator as intermediate trait outputs, and the time to bud outgrowth 147 (days) as the final trait output. The levels of the intermediate trait outputs are described by 148 differential equations, which each contained three terms: (i) a synthesis term, (ii) an interaction 149 term and (iii) a degradation term. Bertheloot et al. (2020) applied a grid search approach, using 150 observed times to bud outgrowth and levels of cytokinins, to parameterize the coefficients of the 151 differential equations.

\section{Description of the in-silico Gene-to-Phenotype Shoot Branching Network}

To quantify the response to selection for time to bud outgrowth in a breeding population,

156 a G2P shoot branching network model was developed. The G2P network connected phenotypic 157 variation within the shoot branching network to allelic variation across a simulated genome. The 158 simulated genomes of the individuals within a reference population of genotypes (Cooper et al., 159 2020) consisted of a single chromosome with 40 causal genetic loci. Each intermediate trait 
160 received additive genetic effects $(\boldsymbol{u})$ from 10 non-pleiotropic causaal genetic loci. The magnitudes

161 of $u$ were sampled from a normal distribution, but the sum of their effects was constrained so that

162 the additive genetic values $(\boldsymbol{a})$ of individuals were within the range observed in experimental data

163 (Table S4, Bertheloot et al. (8)). The additive genetic values $(\boldsymbol{a})$ ) for each intermediate trait were

164 computed by summing the 10 additive genetic effets effects $\left(\boldsymbol{u}_{\boldsymbol{i}}\right)$ according to the genotypes at

165 the casusal geneti loci of each individual:

166

$$
a_{A U X}=\sum_{i=1}^{10} u_{A U X_{i}} ; a_{S U C}=\sum_{i=1}^{10} u_{S U C_{i}} ; a_{C K}=\sum_{i=1}^{10} u_{C K_{i}} ; a_{S L}=\sum_{i=1}^{10} u_{S L_{i}}
$$

The additive genetic values of individuals replaced the synthesis terms in the differential

169 equations, but all other steps remained unchanged from Bertheloot et al. (2020). We provide the

170 following, adapted equations replacing the synthesis terms with the appropriate breeding values

171 purely for thoroughness and reproducibility. The interaction terms $(\gamma)$ and levels of intermediate

172 traits $(\boldsymbol{g})$ were calculated based on the addtive genetic values $(\boldsymbol{a})$ of individuals as follows:

173

$$
\begin{gathered}
\gamma_{C K_{A U X}}=\frac{1}{1+0.96 \cdot a_{A U X}} \\
\gamma_{C K_{S U C}}=0.25 \cdot \frac{a_{S U C}^{2}}{0.19+a_{S U C}^{2}} \\
\gamma_{S L_{A U X}}=24.89 \cdot \frac{a_{A U X}^{2}}{294.58+a_{A U X}^{2}}
\end{gathered}
$$

$$
\begin{gathered}
g_{A U X}=a_{A U X} \\
g_{S U C}=a_{S U C} \\
g_{C K}=\frac{a_{C K} \cdot \gamma_{C K_{A U X}}+\gamma_{C K_{S U C}}}{0.99} \\
g_{S L}=\frac{a_{S L}+\gamma_{S L_{A U X}}}{0.86}
\end{gathered}
$$


177 Calculated as follows:

$$
\begin{aligned}
& \gamma_{I_{C K}}=\frac{1}{1+1000 \cdot g_{C K}} \\
& \gamma_{I_{S L S U C}}=5.64 \cdot \frac{g_{S L}^{2}}{1+\left[\left(0.00418+7.10 \cdot g_{S U C}^{2}\right) \cdot g_{S L}^{2}\right]} \\
& g_{I}=0.33+\gamma_{I_{S L: S U C}}+\gamma_{I_{C K}}
\end{aligned}
$$

The level of the signal integrator $\left(g_{I}\right)$ was then used to calculate the output trait, time to bud outgrowth $\left(g_{\boldsymbol{B}}\right)$, as well as the calculation of a threshold bud outgrowth trait:

$$
g_{B o}=-2.2+3.5 \cdot g_{I}
$$

$$
\begin{aligned}
\text { Bud Ougrowth, } & g_{\text {Bo }} \leq 8.3 \\
\text { No Bud Outgrowth, } & g_{\text {Bo }}>8.3
\end{aligned}
$$

\section{Description of the in-silico Selection Experiments}

For the in-silico selection experiments, we created an initial reference population of 187 genotypes (RPG) from a single biparental cross, consisting of 1,000 F2 individuals. Phenotypes 188 for time to bud outgrowth $\left(\boldsymbol{y}_{\boldsymbol{B}}\right)$ of the 1,000 individuals of the reference population of genotypes 189 were generated by adding a random error effect $\left(e \sim N\left[\mathbf{0}, v_{e}\right]\right)$, to collectively represent stochastic 190 environmental, developmental noise and measurement error, to the true genetic value of time to 191 bud outgrowth, $\boldsymbol{g}_{\boldsymbol{B} \boldsymbol{o}}$ (Eqn. 1). The value of $v_{\boldsymbol{e}}$ was calculated such that the broad-sense 192 heritability, $\boldsymbol{H}^{2}$, of time to bud outgrowth ranged between 0.1 and 1.0 in the initial RPG. We 193 present results for $\boldsymbol{H}^{2}=0.3$ and 1.0. The value of $v_{e}$ was held constant over the 30 selection 194 cycles. Therefore, individuals experienced the same level of random error throughout the 
195 experiment, while the $\boldsymbol{H}^{2}$ for time to bud outgrowth could change with the magnitude of genetic

196 variance in the reference population of genotypes due to selection. The population underwent 30

197 cycles of truncation selection with discrete, non-overlapping generations (cycles) for either higher

198 or lower time to bud outgrowth. For each cycle, the 'best' 100 individuals were selected based on

199 their output trait phenotype, time to bud outgrowth, to be used as parents (selection proportion =

200 0.1) and crossed at random to create 1,000 offspring for the next cycle of evaluation and

201 selection. Independent population replicates were generated by repeating the whole in-silico

202 selection experiment process one hundred times.

Hierarchical Clustering of Selection Trajectories

206 Multi-trait performance landscapes for the intermediate trait phenotypes were generated to 207 investigate quantitative genetic properties of the selection trajectories over 30 cycles of selection

208 (Gavrilets, 2004; Messina et al., 2011; Walsh and Lynch, 2018; Cooper et al., 2020). To aid the 209 visualization of the exploration of the shoot branching performance landscape via selection, the 210 selection trajectories of the 100 replicates underwent hierarchical clustering. The 100 replicates 211 were classified into 3 clusters using Ward's method (Ward, 1963; Wishart, 1969; Williams, 1976).

212 Rows of the matrix corresponded to the replicate id for each of the 100 replicates, columns of the 213 matrix corresponded to each of the 30 selection cycles, and the cells contained values for 214 strigolactone levels. The group mean trait levels for each selection cycle were plotted on the 215 performance landscapes to visualize the selection trajectories. 


\section{Results}

The in-silico selection experiments with the shoot branching G2P network (Fig. 1)

219 revealed the presence of cryptic genetic variation for the intermediate traits, hormones, and

220 sugars that the selection for time to bud outgrowth struggled to access (Waddington, 1942; Flatt,

221 2005; Masel, 2006; Walsh and Lynch, 2018). Such cryptic sources of genetic variation occur

222 when selection cannot directly translate the sources of genetic variation into a selection response

223 to improve adaptation and performance. The cryptic genetic variation within the in-silico

224 experiment led to many repeated selection cycles with reduced selection response and large

225 variation in selection trajectories across different replicate populations (Fig. $2 A, C$ ). Despite the

226 large magnitudes of cryptic genetic variation for the intermediate traits under indirect selection,

227 only small differences were observed among the selection trajectories of time to bud outgrowth,

228 the output trait under direct selection.

229 Cryptic genetic variation for the intermediate traits resulted in temporary and permanent 230 plateaus in selection response (Fig. $2 A, C$ ). The emergence of these plateaus began after only 231 three selection cycles. In scenarios with a simulated broad-sense heritability of 1 (no stochastic 232 error variation during selection), the average genetic mean for sucrose began to increase again 233 around selection cycle nine, when the genetic mean for strigolactones reached approximately 0.1 . 234 However, even with this perfect selection accuracy, a few of the replicate populations reached 235 permanent plateaus at local maxima for two intermediate traits, sucrose and strigolactones. In 236 scenarios with error included in the phenotypes of time to bud outgrowth, selection response was 237 decreased for all shoot branching G2P network components, with the largest reductions observed 238 for the genetic mean of sucrose. For example, with a broad-sense heritability of 0.3 , the average 239 genetic mean for sucrose across the 100 population replicates reached a permanent plateau at 240 less than $90 \%$ of the maximum theoretical value after 30 selection cycles (Fig. 2C), with several 241 individual populations achieving less than $60 \%$. 
242 Different magnitudes of cryptic genetic variation across the intermediate traits of 243 hormones and sucrose resulted in different allele frequency changes for causal loci with similar 244 genetic effect sizes for the intermediate traits (Fig. 2B, D). This trend was most apparent for 245 causal loci occupying the bottom $60 \%$ of genetic effect sizes. In this G2P network simulation, 246 alleles of causal loci with moderate genetic effect sizes $(0.2-0.6)$ for cytokinins and auxin reached 247 fixation within the reference population of genotypes (allele frequency of 0 or 1 ). However, causal 248 loci for sucrose and strigolactones, also with moderate genetic effect sizes, were still segregating 249 in the reference population of genotypes after 30 selection cycles (Fig. 2D). In the most extreme 250 cases of the population replicates, causal loci with small genetic effect sizes $(<0.2)$ underwent 251 genetic drift in the reference population of genotypes, with allele frequency changes in the 252 opposite direction from that expected based on the direction of selection. For example, we 253 observed increases in allele frequencies of causal loci for strigolactones until at least selection 254 cycle 10 (Fig. 2B, D) even though, in the absence of genetic drift or interaction effects, these 255 would be expected to be selected against under the direct selection for faster bud outgrowth (Fig. 256 1). This property of intermediate traits was independent of the heritability of the trait under direct 257 selection.

258 Performance landscapes were generated to further investigate and visualize emergent 259 properties at the intermediate levels of the shoot branching network (Fig. 3). Steep gradients for 260 time to bud outgrowth were observed at intermediate values of the intermediate traits, 261 strigolactones and sucrose. Flatter gradients for time to bud outgrowth values were observed at 262 extreme values of the intermediate traits. The plateaus in the performance landscape reflect that 263 the large sources of genetic variation for sucrose and strigolactones translated into only a small 264 variation in values of time to bud outgrowth. The average selection trajectories of the population 265 replicates followed the steepness of performance landscapes, with a consistent higher strength of 266 selection for higher sucrose levels in the first few selection cycles, followed by selection for lower 267 strigolactone levels (Fig 3A \& 3B). Although there was considerable variability in the selection 268 trajectories among the individual population replicates (Supplementary Fig. 1). The inclusion of 
bioRxiv preprint doi: https://doi.org/10.1101/2022.02.03.479064; this version posted February 5, 2022. The copyright holder for this preprint

(which was not certified by peer review) is the author/funder, who has granted bioRxiv a license to display the preprint in perpetuity. It is made available under aCC-BY-NC-ND 4.0 International license.

269 stochastic error in time to bud outgrowth values, $H^{2}=0.3$, resulted in selection trajectories

270 stopping at a local maximum instead of the global maximum (Fig. 3B).

271 


\section{Discussion}

In this study, cryptic genetic variation (Waddington, 1942; Masel, 2006; Walsh and Lynch,

275 2018) accumulated over selection cycles for the intermediate traits (hormones and sucrose) of

276 the shoot branching G2P network, which resulted in reduced selection responses. The cryptic

277 genetic variation for sucrose can be explained by the complex interaction between sucrose and

278 strigolactone signalling in the G2P network (Fig. 1), resulting in genotypes with completely

279 different combinations of strigolactone and sucrose levels producing similar values for time to bud

280 outgrowth (Fig. 4). The occurrence of multiple intermediate G2P states mapping to fewer output

281 trait states, associated with the emergent quantitative genetic property of cryptic genetic variation

282 identified for the branching network model (Fig. 1), complicates the prediction of phenotype from

283 genotype and the outcomes of selection strategies (Fig. 2 \& 3), as implemented in plant breeding 284 programs.

285 The accumulation of cryptic genetic variation is not specific to the shoot branching G2P 286 network. It can occur whenever non-linear relationships exist among traits or casual genetic loci 287 due to genetic canalization (Waddington, 1942; Flatt, 2005). Therefore, we expect genetic 288 canalization to be pervasive in complex traits under selection that result from interactions among 289 multiple interacting genes (Kauffman et al., 2004; Rünneburger and Le Rouzic, 2016; Ødegård 290 and Meuwissen, 2016), traits, environments, and agronomic management practices. The 291 expectation of the emergent property of genetic canalization for G2P networks controlling 292 complex traits raises several important questions for crop improvement programs.

293 What are the impacts of reductions in detectable genetic variation for complex traits 294 under selection? Reductions in detectable genetic variation, in part due to genetic canalization, 295 have important implications for the selection responses achieved by crop improvement programs. 296 Reductions in detectable genetic variation for complex target traits reduce the accuracy of 297 identifying the best performing individuals, which leads to plateaus in selection response (Fig. 2A, 298 2C, 3B). Of even more concern, the combination of reduced accuracy and reduced detectable 
299 genetic variation could result in mistaking plateaus associated with local maxima for true, 300 permanent selection limits for breeding populations (Fig. 3B).

301 The results obtained from the in-silico selection experiment based on the branching 302 network (Fig. 1) highlight an important G2P prediction question for plant breeders; How can crop 303 improvement programs promote decanalization? Decanalization would allow the release of 304 cryptic genetic variation for complex traits and accelerate selection response for the reference 305 population of genotypes. In this study, decanalization and the subsequent increases in selection 306 response occurred via the fixation of causal loci by chance (genetic drift) at different cycles for 307 different population replicates (Fig 2B, D). Similar decanalization events could be a contributing 308 factor to the unexpected (according to an additive finite locus model and the breeder's equation; 309 (Falconer and Mackay, 1996; Walsh and Lynch, 2018)), continued selection responses seen in 310 long-term selection experiments (Dudley, 2007; Goodnight, 2015). A more targeted strategy 311 would be to restructure crop breeding programs to promote and control the conversion of non312 additive, epistatic genetic variance into additive genetic variance within the reference population 313 of genotypes (Cooper et al., 2005). For example, in maize (Zea mays), Technow et al. (2021) 314 demonstrated that a decentralized structure of multiple, smaller crop improvement programs 315 interconnected by a few key parents was required to facilitate selection response under high 316 levels of G2P genetic complexity. Another complementary strategy involves direct selection on 317 traits at intermediate layers of the G2P network to circumvent the complex interactions that 318 generate canalization of traits at higher levels of the network hierarchy. In the case of shoot 319 branching, decanalization could be achieved by direct selection on sucrose or strigolactone 320 levels. The many influences of such emergent non-linear properties and the need to consider 321 alternative breeding strategies to accelerate improvement of the complex traits motivate 322 experimental-simulation investigations to develop appropriate G2P models (Marshall-Colon et al., 323 2017; Hammer et al., 2019; Cooper et al., 2020; Tardieu et al., 2020)

324 The development of G2P networks that link, empirically based, plant models with natural 325 genomic variation is crucial to appropriately design strategies and experiments to tackle many 
326 compelling questions in crop improvement. This study is a demonstration of the ideas of

327 modelling selection response through crop growth models outlined in Cooper et al. (2020), using

328 the plant branching network model developed by Bertheloot et al. (2020). A benefit of taking such

329 a view of the G2P relationship was the observation of genetic canalization as an emergent

330 property of selection for faster shoot branching, which would not have been achievable taking

331 standard physiological modelling or single-trait quantitative genetics approaches in isolation. The

332 multi-trait structure and interactions within plant models generate conditional effects that

333 contribute to the quantitative genetic properties of epistasis and vertical pleiotropy when

334 connected to genomic variation. Specifying the genetic effects of the inputs of plant models at the

335 level of genes instead of genotypes allows the assessment of selection response over multiple

336 selection cycles, as is required for the design of breeding strategies (Hammer et al., 2019;

337 Cooper et al., 2020). Such G2P networks also include genetic constraints enforced by processes

338 such as recombination and linkage to provide more realistic predictions of the exploration of

339 performance landscapes of traits (Messina et al., 2011; Technow et al., 2021). In this study,

340 selection for faster shoot branching explored a relatively small area of the total performance

341 landscape encoded by the Bertheloot et al. (2020) branching model (Fig. 3).

Future studies can exploit the increased power and flexibility when viewing complex traits

343 as gene-to-phenotype networks in: (i) in silico simulations (Hammer et al., 2019; Cooper et al.,

344 2020), akin to our approach; (ii) empirical, longitudinal, "select and sequence" studies (Lenski and

345 Travisano, 1994; Wisser et al., 2019) or (iii) broader exploration of performance landscapes with

346 genome editing of network components (Eshed and Lippman, 2019) to improve understanding of

347 selection response of complex traits in nature and agriculture. 


\section{Acknowledgements}

349 We thank Dr Franziska Fichtner for fruitful discussions about the molecular physiology of the

350 shoot branching network and comments on the manuscript. Figures were created or formatted

351 with Biorender.

352

\section{Author Contributions}

354 OMP and MC conceived the study and helped interpret the results. OMP developed the gene-to-

355 phenotype network, developed the in-silico selection experiments, performed the analyses, and

356 wrote the manuscript. FB provided input on the shoot branching model, helped interpret the

357 results, and wrote parts of the introduction. KVF developed a previous iteration of the G2P

358 network. CB provided input on the shoot branching model and helped interpret the results. All

359 authors read, refined, and approved the final manuscript.

\section{Funding Statement}

362 Contribution supported by the Australian Research Council Centre of Excellence for Plant

363 Success in Nature and Agriculture (CE200100015) (OMP, FB, CB and MC), the Australian

364 Grains Research and Development Corporation project UOQ1903-008RTX (OMP and MC), the

365 Australian Research Council Georgina Sweet Laureate Fellowship (FL180100139 to CB) and the

366 Australian Research Council Discovery Early Career Researcher Award (DE210101407 to KVF)

\section{Competing Interest Statement}

368 The authors declare they have no competing interests. 
Barbier, F. F., Dun, E. A., Kerr, S. C., Chabikwa, T. G., and Beveridge, C. A. (2019). An Update on the Signals Controlling Shoot Branching. Trends Plant Sci 24, 220-236. doi:10.1016/j.tplants.2018.12.001.

373 Benfey, P. N., and Mitchell-Olds, T. (2008). From Genotype to Phenotype: Systems Biology

374 Meets Natural Variation. Science 320, 495. doi:10.1126/science.1153716.

375 Bertheloot, J., Barbier, F., Boudon, F., Perez-Garcia, M. D., Péron, T., Citerne, S., et al. (2020). 376 Sugar availability suppresses the auxin-induced strigolactone pathway to promote bud outgrowth. 377 New Phytologist 225, 866-879. doi:https://doi.org/10.1111/nph.16201.

378 Cooper, M., Messina, C. D., Podlich, D., Totir, L. R., Baumgarten, A., Hausmann, N. J., et al. 379 (2014). Predicting the future of plant breeding: complementing empirical evaluation with genetic 380 prediction. cpsc 65, 311-336. doi:10.1071/CP14007.

381 Cooper, M., Podlich, D. W., and Smith, O. S. (2005). Gene-to-phenotype models and complex 382 trait genetics. Aust. J. Agric. Res. 56, 895-918. doi:10.1071/AR05154.

Cooper, M., Powell, O., Voss-Fels, K. P., Messina, C. D., Gho, C., Podlich, D. W., et al. (2020). Modelling selection response in plant breeding programs using crop models as mechanistic geneto-phenotype (CGM-G2P) multi-trait link functions. in silico Plants.

doi:10.1093/insilicoplants/diaa016.

387 Domagalska, M. A., and Leyser, O. (2011). Signal integration in the control of shoot branching.

388 Nature Reviews Molecular Cell Biology 12, 211-221. doi:10.1038/nrm3088.

389 Dudley, J. W. (2007). From Means to QTL: The Illinois Long-Term Selection Experiment as a

390 Case Study in Quantitative Genetics. Crop Science 47, S-20-S-31.

391 doi:https://doi.org/10.2135/cropsci2007.04.0003IPBS.

392 Eshed, Y., and Lippman, Z. B. (2019). Revolutions in agriculture chart a course for targeted 393 breeding of old and new crops. Science 366, eaax0025. doi:10.1126/science.aax0025.

394 Falconer, D. S., and Mackay, T. F. C. (1996). Introduction to Quantitative Genetics. Harlow, UK: 395 Longman.

396 Fichtner, F., Dissanayake, I. M., Lacombe, B., and Barbier, F. (2021). Sugar and Nitrate Sensing: 397 A Multi-Billion-Year Story. Trends Plant Sci 26, 352-374. doi:10.1016/j.tplants.2020.11.006.

398 Flatt, T. (2005). The Evolutionary Genetics of Canalization. The Quarterly Review of Biology 80, 399 287-316. doi:10.1086/432265.

400 Gavrilets, S. (2004). Fitness Landscapes and the Origin of Species. Available at:

401 https://press.princeton.edu/books/paperback/9780691119830/fitness-landscapes-and-the-origin402 of-species-mpb-41 [Accessed January 28, 2022].

403 Gianola, D. (2021). Opinionated Views on Genome-Assisted Inference and Prediction During a 404 Pandemic. Frontiers in Plant Science 12, 1533. doi:10.3389/fpls.2021.717284. 
405 Goodnight, C. (2015). Long-term selection experiments: epistasis and the response to selection.

406 Methods Mol Biol 1253, 1-18. doi:10.1007/978-1-4939-2155-3_1.

407 Hammer, G., Messina, C., Wu, A., and Cooper, M. (2019). Biological reality and parsimony in

408 crop models - why we need both in crop improvement! in silico Plants 1, diz010.

409 doi:10.1093/insilicoplants/diz010.

410 Kauffman, S., Peterson, C., Samuelsson, B., and Troein, C. (2004). Genetic networks with 411 canalyzing Boolean rules are always stable. PNAS 101, 17102-17107.

412 doi:10.1073/pnas.0407783101.

413 Kholová, J., Urban, M. O., Cock, J., Arcos, J., Arnaud, E., Aytekin, D., et al. (2021). In pursuit of a 414 better world: crop improvement and the CGIAR. Journal of Experimental Botany 72, 5158-5179.

415 doi:10.1093/jxb/erab226.

416 Lenski, R. E., and Travisano, M. (1994). Dynamics of adaptation and diversification: a 10,000-

419 Marjoram, P., Zubair, A., and Nuzhdin, S. V. (2014). Post-GWAS: where next? More samples, 420 more SNPs or more biology? Heredity 112, 79-88. doi:10.1038/hdy.2013.52.

421 Marshall-Colon, A., Long, S. P., Allen, D. K., Allen, G., Beard, D. A., Benes, B., et al. (2017).

422 Crops In Silico: Generating Virtual Crops Using an Integrative and Multi-scale Modeling Platform.

423 Frontiers in Plant Science 8. Available at:

424 https://www.frontiersin.org/article/10.3389/fpls.2017.00786 [Accessed January 28, 2022].

425 Masel, J. (2006). Cryptic Genetic Variation Is Enriched for Potential Adaptations. Genetics 172, 426 1985-1991. doi:10.1534/genetics.105.051649.

427 Mason, M. G., Ross, J. J., Babst, B. A., Wienclaw, B. N., and Beveridge, C. A. (2014). Sugar 428 demand, not auxin, is the initial regulator of apical dominance. Proc Natl Acad Sci U S A 111, 429 6092-6097. doi:10.1073/pnas.1322045111.

430 Messina, C. D., Podlich, D., Dong, Z., Samples, M., and Cooper, M. (2011). Yield-trait 431 performance landscapes: from theory to application in breeding maize for drought tolerance.

432 Journal of Experimental Botany 62, 855-868. doi:10.1093/jxb/erq329.

433 Meuwissen, T. H. E., Hayes, B. J., and Goddard, M. E. (2001). Prediction of Total Genetic Value

439 Paixão, T., and Barton, N. H. (2016). The effect of gene interactions on the long-term response to 440 selection. Proc. Natl. Acad. Sci. U.S.A. 113, 4422-4427. doi:10.1073/pnas.1518830113.

441 Patil, S. B., Barbier, F. F., Zhao, J., Zafar, S. A., Uzair, M., Sun, Y., et al. (2021). Sucrose 442 promotes D53 accumulation and tillering in rice. New Phytologist. doi:10.1111/nph.17834. 
443 Reynolds, M., Atkin, O. K., Bennett, M., Cooper, M., Dodd, I. C., Foulkes, M. J., et al. (2021).

444 Addressing Research Bottlenecks to Crop Productivity. Trends in Plant Science 26, 607-630.

445 doi:10.1016/j.tplants.2021.03.011.

446 Rünneburger, E., and Le Rouzic, A. (2016). Why and how genetic canalization evolves in gene

447 regulatory networks. BMC Evolutionary Biology 16, 239. doi:10.1186/s12862-016-0801-2.

448 Salam, B. B., Barbier, F., Danieli, R., Ziv, C., Spíchal, L., Teper-Bamnolker, P., et al. (2020).

449 Sucrose promotes etiolated stem branching through activation of cytokinin accumulation followed

450 by vacuolar invertase activity. bioRxiv, 2020.01.08.897009. doi:10.1101/2020.01.08.897009.

451 Tardieu, F., Granato, I. S. C., Van Oosterom, E. J., Parent, B., and Hammer, G. L. (2020). Are

452 crop and detailed physiological models equally "mechanistic" for predicting the genetic variability

453 of whole plant behaviour? - the nexus between mechanisms and adaptive strategies. in silico

454 Plants. doi:10.1093/insilicoplants/diaa011.

455 Technow, F., Podlich, D., and Cooper, M. (2021). Back to the future: implications of genetic 456 complexity for the structure of hybrid breeding programs. G3 Genes/Genomes/Genetics 11. 457 doi:10.1093/g3journal/jkab153.

458 Waddington, C. H. (1942). Canalization of develpoment and genetic assimilation of acquired 459 characters. Nature 150, 563-565. doi:10.1038/150563a0.

460 Walsh, B., and Lynch, M. (2018). Evolution and Selection of Quantitative Traits. OUP Oxford.

461 Ward, J. H. (1963). Hierarchical Grouping to Optimize an Objective Function. Journal of the

462 American Statistical Association 58, 236-244. doi:10.1080/01621459.1963.10500845.

463 Williams, W. T. (1976). Pattern analysis in agricultural science. Elsevier.

464 Wishart, D. (1969). An algorithm for hierarchical classification. Biometrics 22, 165-170.

465 Wisser, R. J., Fang, Z., Holland, J. B., Teixeira, J. E. C., Dougherty, J., Weldekidan, T., et al. 466 (2019). The Genomic Basis for Short-Term Evolution of Environmental Adaptation in Maize.

467 Genetics 213, 1479-1494. doi:10.1534/genetics.119.302780.

468 Wu, A., Hammer, G. L., Doherty, A., von Caemmerer, S., and Farquhar, G. D. (2019). Quantifying 469 impacts of enhancing photosynthesis on crop yield. Nat. Plants 5, 380-388. doi:10.1038/s41477470 019-0398-8. 
bioRxiv preprint doi: https://doi.org/10.1101/2022.02.03.479064; this version posted February 5, 2022. The copyright holder for this preprint (which was not certified by peer review) is the author/funder, who has granted bioRxiv a license to display the preprint in perpetuity. It is made available under aCC-BY-NC-ND 4.0 International license.

472

473

474

475

476

477

478

479
Figures

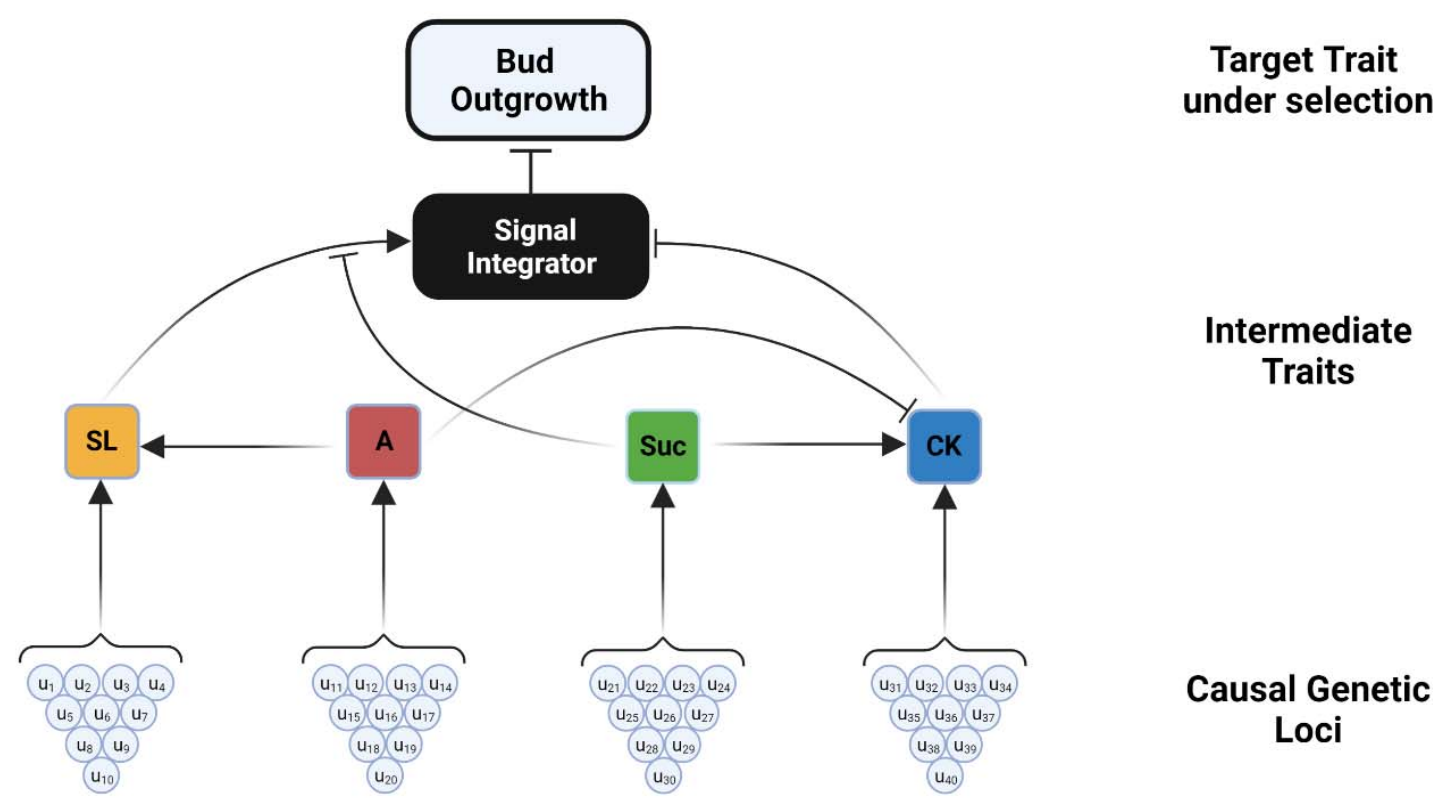

Figure 1. Shoot branching gene-to-phenotype (G2P) network. Ten causal genetic loci (u) and interactions determine the levels of each of the intermediate traits : strigolactones (SL), auxin (A), sucrose (Suc), and cytokinins (CK). In turn, levels of these intermediate traits (hormones, sucrose and the signal integrator), their interactions, and random error (e) determine the time to bud outgrowth of an individual plant. 


\section{Selection For Faster Bud Outgrowth}
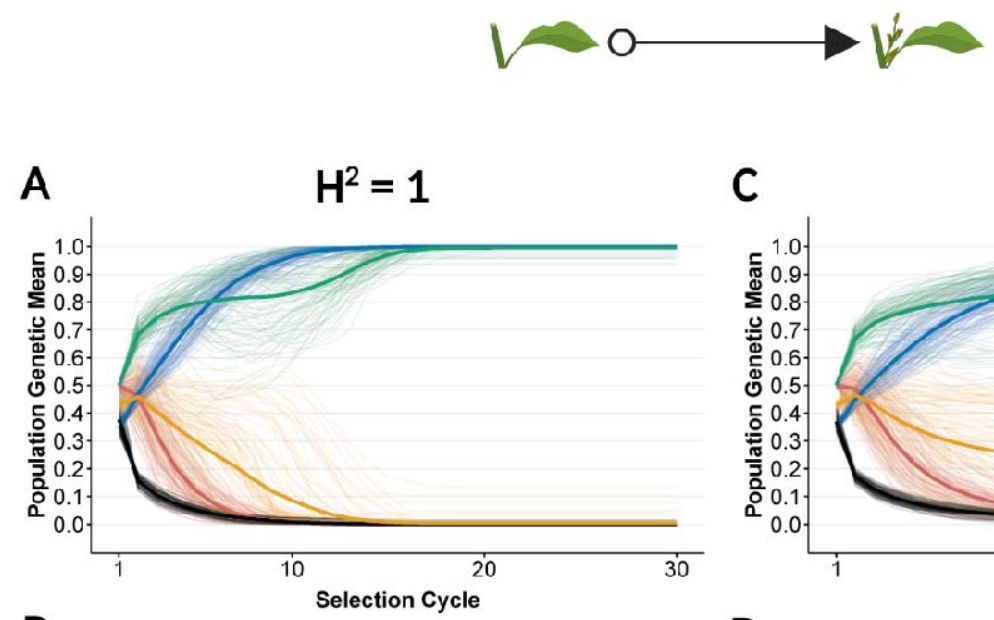

B
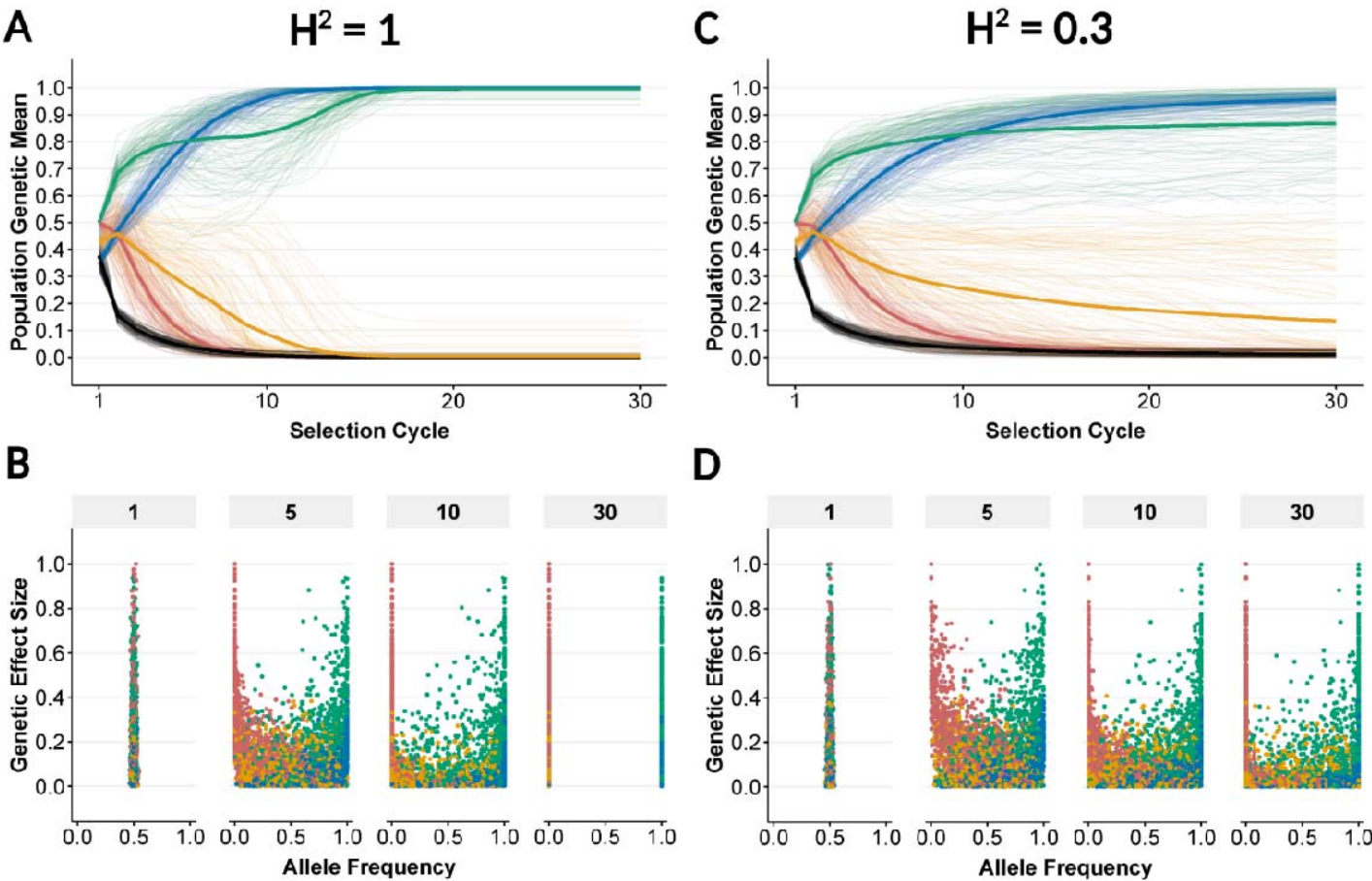

D

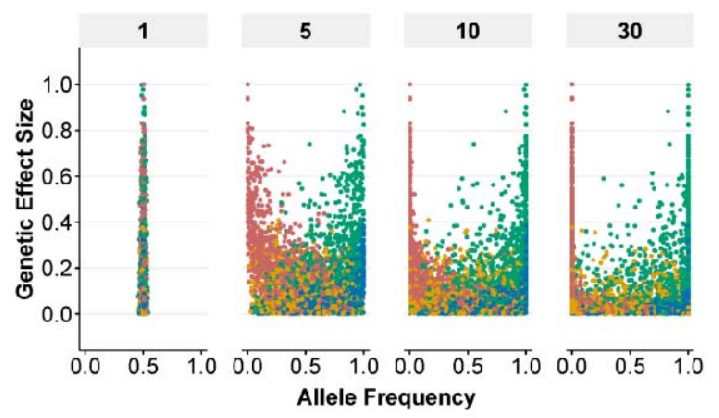

Intermediate Traits

Sucrose

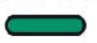

Cytokinins

Signal Integrator

Figure 2. Selection trajectories for the shoot branching G2P network for faster bud outgrowth and the relationships between normalized genetic effect sizes and allele frequency changes at causal genetic loci over selection cycles. $(A, B)$ Results from selection with a broad sense heritability, , of 1 . $(\boldsymbol{C}, \boldsymbol{D})$ Results from selection with a broad sense heritability, , of 0.3 . $(\boldsymbol{A}, \boldsymbol{C})$ Normalized total genetic values for the intermediate traits over selection cycles. Thick lines are the normalized genetic means averaged across the population replicates. Thin lines are the normalized genetic means for each population replicate. $(\boldsymbol{B}, \boldsymbol{D})$ Plot of allele frequency changes at causal genetic loci versus normalized genetic effect sizes. Each point represents a causal genetic locus from the 100 population replicates. Values are presented for selection cycles $1,5,10$, and 30 . 


\section{Selection For Faster Bud Outgrowth}
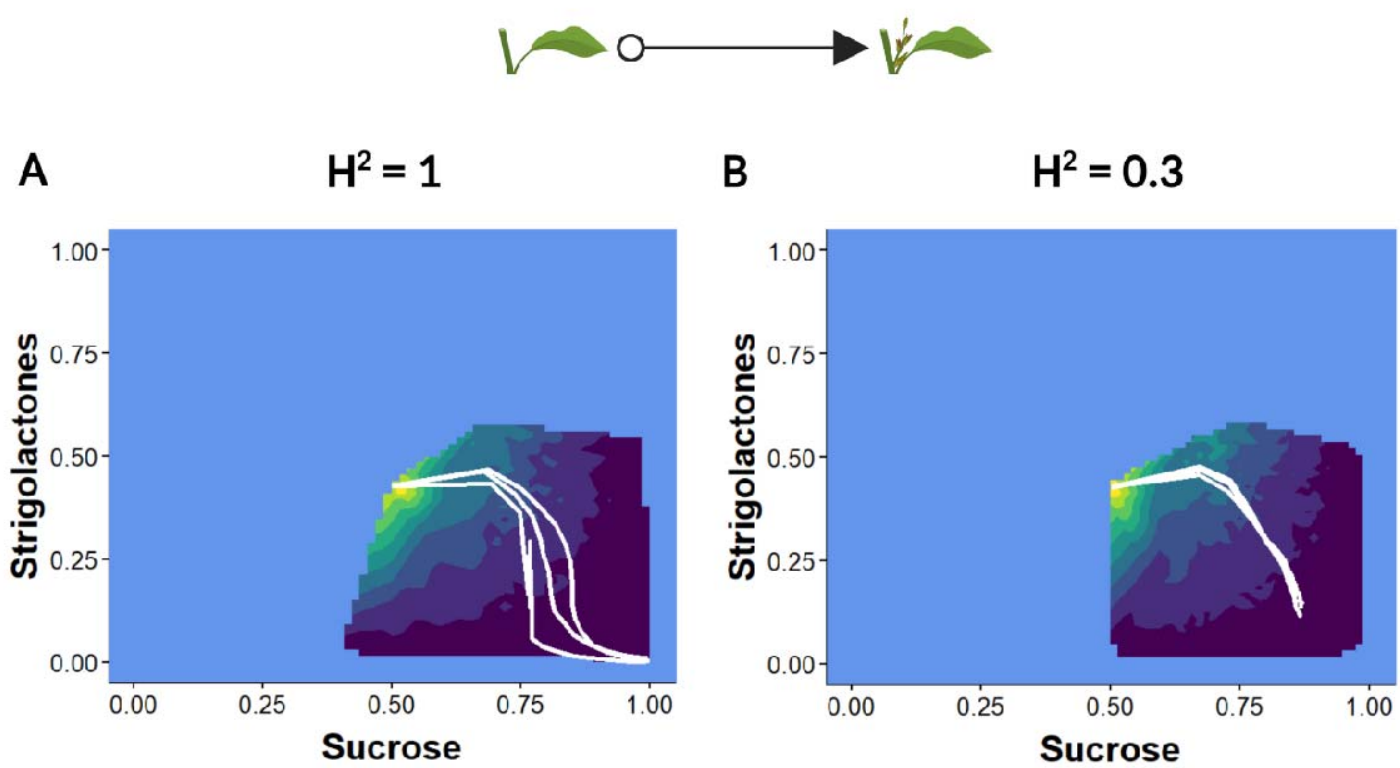

492

\section{Figure 3. Exploration of the Shoot Branching Performance Landscape via Selection. $(A)$} Results from selection with a broad sense heritability, , of 1. (B) Results from selection with a broad sense heritability, , of 0.3 . To aid visualization, the selection trajectories of 100 population replicates were grouped into 3 clusters using Ward's method (see Methods). The average selection trajectories of the three clusters (thick white lines) are plotted against the normalised total genetic values for strigolactones and sucrose. The contour shows the variation in strigolactone and sucrose values and the values for time to bud outgrowth observed across the 100 population replicates. The light blue area depicts the area of the full shoot branching performance landscape, determined by the Bertheloot et al. (2020) model, unexplored by selection. 
bioRxiv preprint doi: https://doi.org/10.1101/2022.02.03.479064; this version posted February 5, 2022. The copyright holder for this preprint (which was not certified by peer review) is the author/funder, who has granted bioRxiv a license to display the preprint in perpetuity. It is made available under aCC-BY-NC-ND 4.0 International license.

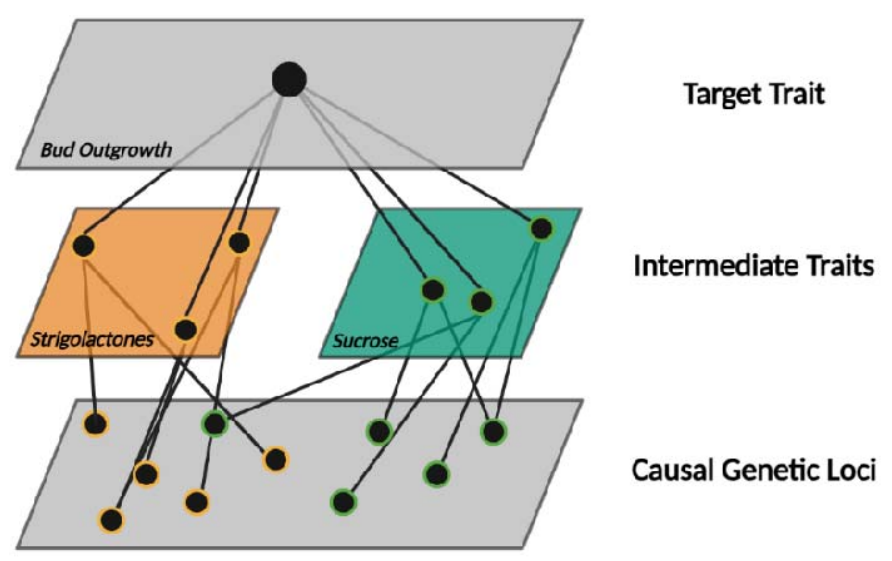

504

Figure 4. Genetic Canalization of Gene-To-Phenotype Networks. Multiple genetic combinations of intermediate traits produce similar values for the target trait, causing the accumulation of cryptic genetic variation that cannot be accessed by selection.

508 


\section{Selection For Faster Bud Outgrowth}
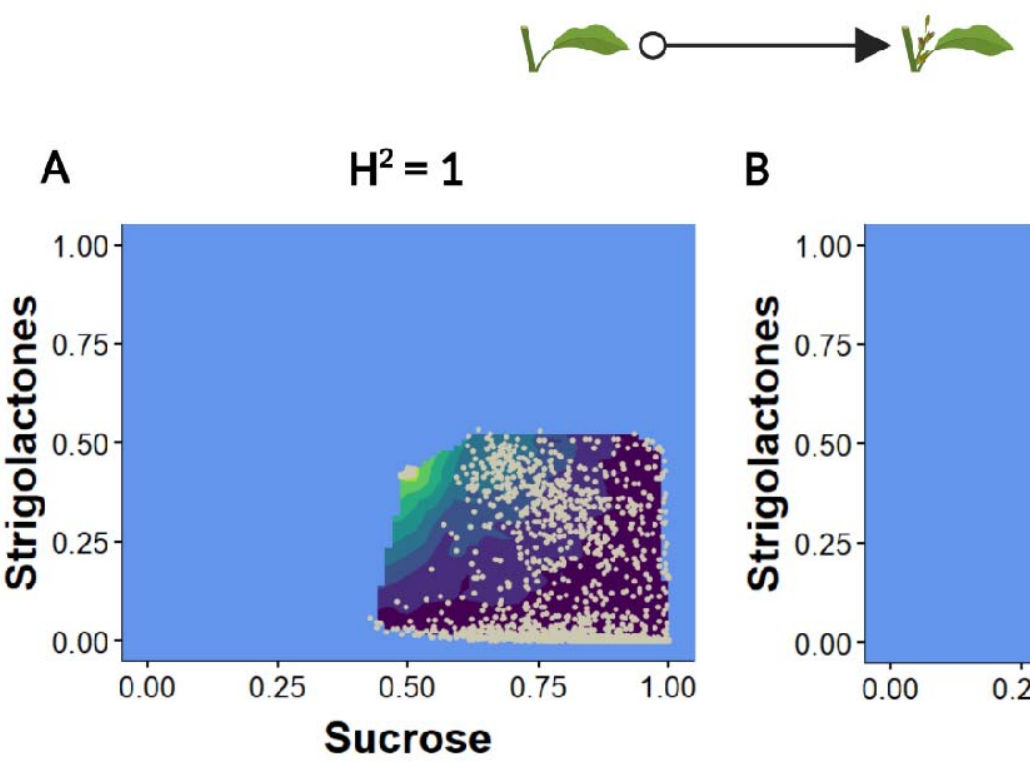

B $\quad H^{2}=0.3$

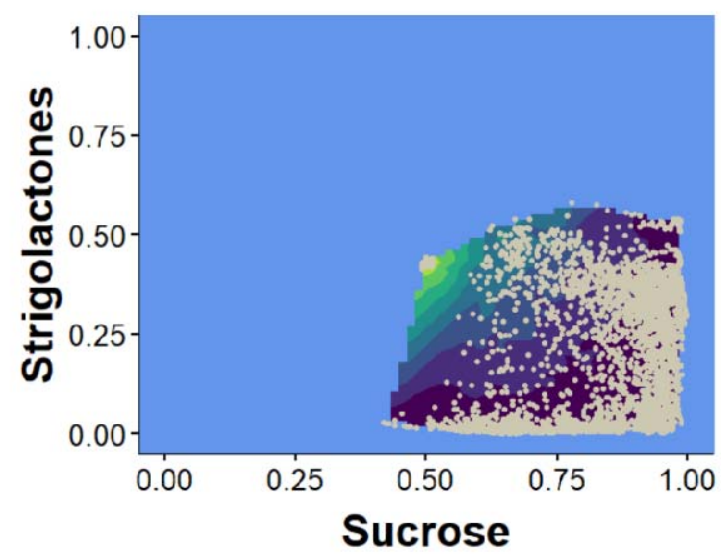

Supplementary Figure 1. Population variation during the exploration of the shoot branching performance landscape. (A) Results from selection with a broad sense heritability,

, of 1. (B) Results from selection with a broad sense heritability,

, of 0.3. The contour shows the variation in strigolactone and sucrose values and the values for time to bud outgrowth observed across the 100 population replicates. The light blue area depicts the area of the full shoot branching performance landscape, determined by the Bertheloot et al. (2020) model, unexplored by selection. Grey dots show average strigolactone and sucrose values for population replicates at a particular selection cycles. 